\title{
Innovation and integration at the Warren Alpert Medical School of Brown University
}

\author{
Edward J. Wing', Richard Dollase ${ }^{2}$, Luba Dumenco ${ }^{3}$, Paul George ${ }^{4}$
}

$\mathrm{A}$ $\mathrm{s}$ Dean of Medicine and Biological Sciences for Brown University from 2008-2013, I oversaw a curriculum that reflects the core of what I believe are the essential components of a medical student's education. This article describes the philosophy and content of that curriculum.

\section{INTRODUCTION}

The curriculum of the Warren Alpert Medical School of Brown University (Alpert Medical School or AMS) is patient centered, competency based, integrated, and continuously evolving. There are many innovative aspects of the curriculum that reflect the overall culture at Brown University and the fact that the medical school is relatively new, graduating its first class in 1975. The curriculum is based on the following principles:

1. Teaching and evaluation are competency based;

2. The curriculum is centrally controlled;

3. The preclinical curriculum is integrated;

4. Clinical skills are taught in all four years;

5. There is an opportunity for scholarship and research in all four years;

6. There is an emphasis on creating a supportive environment for students;

7. Curriculum innovation is fostered and valued.
The success of the curriculum has been demonstrated by the quality of the medical students that are admitted, their satisfaction with their education, their objective test scores, and the quality of the residencies the students are accepted into after graduation. Descriptions of the AMS curriculum and newer initiatives have been published ${ }^{1-6}$.

\section{Competency-Based Curriculum}

A competency-based curriculum was introduced at AMS in 2000-01 making it the first medical school in the United States to establish competency standards and assessment protocols to evaluate student progress. The program was revised and retitled The Nine Abilities in 2011. The Nine Abilities are:

1. Effective Communication;

2. Basic Clinical Skills;

3. Using Basic Science in the Practice of Medicine;

4. Diagnosis, Prevention and Treatment;

5. Lifelong Learning;

6. Professionalism;

7. Community Health Promotion and Advocacy;

8. Moral Reasoning and Clinical Ethics;

9. Clinical Decision Making.

A more complete description of the abilities can be found in Reference \#1.

1. MD, Professor of Medicine, The Warren Albert Medical School of Brown University. Providence, Rhode Island.

2. Ed.D, Director, Office of Medical Education, The Alpert Medical School of Brown University. Providence, Rhode Island.

3. MD, Director, Preclinical Curriculum, The Alpert Medical School of Brown University, Providence, Rhode Island.

4. Director, Year Two Curriculum, Director, Primary Care-Population Medicine Program/Longitudinal Integrated Clerkship, Office of Medical Education, Associate Professor, Department of Family Medicine, Associate Professor, Section on Medical Education, The Warren Alpert Medical School of Brown University, Providence, Rhode Island. 
Curriculum objectives and planning are based on these competencies, and assessments of students by faculty are based on the achievement of the abilities in the targeted area. Achievement of abilities is measured not only by faculty assessment but also by written examinations and Objective Structured Clinical Examinations (OSC-Es).

\section{Central Oversight and Control}

Responsibility and control of the curriculum rests with the Dean of AMS who typically delegates this task to the Associate Dean for Medical Education and the Medical Curriculum Committee. Under the Associate Dean is a team of curriculum specialists and physicians dedicated to the education of the medical students. This group of medical educators is ultimately responsible for the strategy, quality, quantity, assessment and improvement of the educational experience. They are also responsible for fulfilling national accreditation standards and university regulations and requirements. Naturally, they must work closely with faculty and students to plan and evaluate the curriculum. In fact, one of the most important characteristics of this group of educators at Brown is their commitment to continuously monitor and improve the course material and delivery. For example, after the end of each course, students evaluate the effectiveness of the teaching and course content in numerical and written responses; in addition, students are brought together in focus groups to evaluate and critique the course and offer suggestions for improvement. This information is fed back to faculty by the educational team who then work with the faculty to improve the material and teaching approach. This type of evaluation takes place in the first two years and results in a continuously evolving and improving preclinical curriculum.

In the third year, the core clerkships are reviewed annually by the Medical Curriculum Committee, and one of the clerkships undergoes a two-day substantive review on a rotating basis. The review team is composed of an outside reviewer who directs a similar clerkship at another medical school, faculty from other departments at AMS, and fourth year students who have completed the clerkship. The review team reports its findings and recommendations to the clerkship director and his or her department chair, to the Medical Curriculum Committee and to the Dean of the Medical School.

Most important in this model is oversight of the curriculum as a whole by the educational team. Strengths and deficiencies can be assessed in light of the entire curriculum. The primary motive of these medical educators is the optimal education of the medical students. This imperative is in contrast to personal agendas such as professors presenting their own research (fascinating to the individual faculty member, not always relevant to the medical students' education) or professors teaching to fulfill requirements for promotion or salary.

\section{Integrated Preclinical Curriculum}

The principle of an integrated preclinical curriculum has been widely accepted in the United States and was instituted at the AMS in 2006. Underlying the principle is the assumption that specific course material is more comprehensible and thus more easily learned if it is integrated across traditional disciplines with regard to structure and function. For example, in the first semester, students at AMS are introduced in Integrated Medical Sciences (IMS) I to cell biology, cell physiology, biochemistry, immunology and genetics interrelated with gross anatomy and histology in an integrated fashion. Participating faculty come from different disciplines, but all work hard to present the material in an understandable biologically relevant context. The development IMS I courses took a major effort on the part of the educational leadership, faculty from different disciplines, and students at AMS. The curriculum including individual lectures, small group sessions and written material is continuously reviewed and evaluated. In addition to the IMS courses, students take a Health System and Policy Course that deals with population health.

A second example occurs at the beginning of the second semester of the first year in the IMS II courses in the Brain Sciences block. Head and neck anatomy are integrated with neurobiology, brain and behavior, neuropathophysiology, and neuropharmacology. The course is taught by basic anatomists, neuroscientists, neurologists, psychiatrists and neurosurgeons. Similarly, later in the second semester microbiology is integrated with infectious diseases and relevant pharmacology. 
Wing EJ, et al. Innovation and integration at the Warren Alpert Medical. Rev Med (São Paulo). 2016 July-Aug.;95(Special Issue 3):19-23. 
The second year IMS III and IV continues a similar system-based approach in seven separate courses taught by faculty from different disciplines. They include cardiovascular, pulmonary, renal, human reproduction, endocrinology, hematology, and gastroenterology. The courses are supplemented with small group learning and a journal club in each course that introduces the approach to reading the medical literature. At the end of this semester, time is reserved for individual preparation for the United States Medical Licensing Examination (USMLE) Step 1 National Board Examination.

In the first two years, students also have the opportunity to participate in electives such as Healthcare in America, Cancer action and Reflection (CARE), Medical Spanish, and Refugee Health and Advocacy.

\section{Clinical Skills}

Clinical skills are taught in the first two years of medical school through an innovative course called Doctoring. As an introduction to clinical medicine, students in this course are taught physical examination, communication skills as well as interviewing and oral presentation techniques through lectures and small group sessions and practice with standardized patients. Students are also introduced early in the semester to outpatient medicine in the offices of practicing physicians for one half day/week. Students initially shadow the mentor but quickly begin to see the patient on their own during which they have the opportunity to practice their history taking and physical examination skills. Students particularly enjoy these sessions because they see the real world of medicine and partake directly in the clinical care of patients. The Doctoring course continues in the second year where skills, particularly physical examination skills, are stressed and further developed. In addition, clinical problem solving is introduced both in small group sessions and the clinical setting.

At the end of the second year, students participate in a Clinical Skills Clerkship to help prepare for the required third year clerkships. In this three week required course, students are introduced to the electronic medical record. How to access clinically relevant literature and data bases and practice guidelines is demonstrated. Procedures such as placing IVs, giving injections, basic suturing and performing lumbar punctures are practiced in simulated settings. Advanced cardiac life support (ACLS) is also introduced. In addition inter-professional team learning is accomplished through sessions with nursing and pharmacy programs at the University of Rhode Island and Rhode Island College.

In the third year students take six required clinical clerkships including Internal Medicine (12 weeks), Surgery (six weeks), Family Medicine (six weeks), Obstetrics/ Gynecology (six weeks), Pediatrics (six weeks), and an integrated Psychiatry/Neurology (eight weeks). They work as an integral part of medical teams and are given graded responsibility for patient care. They also attend conferences and participate in on call with residents. Both inpatient and outpatient experiences are included in each clerkship except for Family Medicine which is outpatient only. At the end of each rotation, students take a NBME Shelf examination that tests clinical knowledge in that specialty. At the end of the third year, students take an OSCE examination that ensures that they have successfully mastered the nine abilities or competencies in each of the clerkship specialties in order to proceed to the fourth year.

In the fourth year students are required to take a four-week subinternship, often in the area of their interest for residency. Many students take an optional 3 month longitudinal outpatient experience also in an area of their interest. Students also have time to take clinical electives either at Brown (where there are more than 150 electives) or at other institutions. In total, students must complete 80 weeks of clinical rotations, 56 of which are required.

\section{Scholarship}

Students at AMS are encouraged to participate in research and almost $90 \%$ of students are involved in a research project with faculty by the time of graduation. Because research is strongly encouraged at AMS, a number of students take leave to work in laboratories or clinical projects. A large number of projects result in publication by the students and their mentors.

Approximately one third of students participate in the Scholarly Concentration Program, which begins in the first year and extends through all four years of medical 
school. In this program students select a topic of interest and meet with mentors to plan a program. In the summer between the first and second year students undertake a ten week project in their Scholarly Concentration area (this can range from basic science research to curriculum development to an overseas project, to writing projects and clinical experiences. Cross-disciplinary, independent projects with close faculty mentorship are encouraged. Summer Assistantship Funding is available for research projects. Work continues during the second and third year. In the fourth year students submit a scholarly thesis or portfolio. Upon successful completion, students graduate with acknowledgment of completion of the Scholarly Concentration. Examples of concentration areas in the scholarly program include Global Health, Medical Humanities, Health Policy Caring for Underserved Communities, Medical Technology and Innovation, Medical Education, and Women's Reproductive Health.

\section{A Culture of Support}

AMS has a culture of strong support for students academically, personally and professionally. Second year students (content tutors) tutor first year students in understanding content and in studying for examinations. Content tutors are chosen for their academic achievement and are coached on teaching techniques. Similarly, an additional cadre of second year students helps to teach first year students clinical skills. Third and fourth year students tutor second year students on content, clinical skills and on preparation for USMLE Step 1.

In addition, there is support through the Dean for Medical Education and his/her staff within the school. A brand new medical school building has allowed the education staff and students to be in close proximity for advising and counseling. Also, the lecture halls, study rooms, small group seminar rooms, the anatomy suite, and recreational and lounge space are in the same building. This proximity allows close interactions between students, faculty and educators at all levels.

Perhaps most important, the deans and educators as well as students have fostered a culture of mutual support with minimal competition to create a positive attitude towards learning and development that is the envy of other schools. This culture has made AMS one of the most popular medical schools in the country and is reflected in the achievements of the students both during medical school and afterwards.

\section{Innovation - The Primary Care-Population Medicine Program}

AMS has always been known for innovation. The first medical school class composed their own Professional Oath instead of the traditional Hippocratic Oath. It has been repeated at each graduation since then. From a competency based strategy and evaluation system to an integrated curriculum with a robust Doctoring Course and the opportunity to participate in the Scholarly Concentration Program, AMS, its students and leaders, have always shown creativity in the pursuit of outstanding medical education.

The newest example is the Primary Care-Population Medicine Program (PCPM) that began in August, 2015. Primary care is essential to excellence in medical care, but it is estimated that there will be a deficit of more than 30,000 primary care doctors by the year 2035 in the US. Population Medicine refers to the design, delivery, coordination and payment of health care services for a population. The new PCPM program is designed to educate physician leaders in both primary care and population medicine. The students selected for this program have a strong interest and track record in both primary care and health at a population level. Upon graduation, students in the program will be awarded both an MD degree and a Master of Science Degree in Population Medicine. During the first two years, students will take the traditional preclinical curriculum as well as courses in Population Medicine such as Health Systems and Policy, Research Methods and Quantitative Methods, Leadership, and Clinical and Population Medicine. In the third year, instead of traditional clinical clerkships, students in the PCPM will participate in a longitudinal clinical experience. They will be assigned patients from the various specialties to populate a panel that the students will follow for the entire year. For example, they will have patients who are pregnant, patients undergoing surgery, and patients with cardiovascular disease. Over the course of the year, they will have direct experience with their patients in each of the clinical subspecialties. In similar programs at other schools, 
students learn as much, do as well on examinations, and have a more rounded and positive experience compared to students in traditional clerkships. In the fourth year, students in the PCPM program will prepare and present a thesis on Population Medicine.

\section{REFERENCES}

1. Dumenco L, George P, Scott Taylor J, Dollase R. Curriculum innovation at the Warren Alpert Medical School of Brown University. Med Healthy R I. 2012;95(10):317-8,324-7. Available from: http://www. rimed.org/medhealthri/2012-10/2012-10-317.pdf.

2. George P, Tunkel A, Dollase R, Gruppuso P, Dumenco L, Rapoza B, Borkan J. The primary care-population medicine program at the Warren Alpert Medical School of Brown University. R I Med J (2013). 2015;98(9):1621.

3. Mello MJ, Feller E, George P, Borkan J. Advancing the integration of population medicine into medical curricula at the Warren Alpert Medical School of Brown University: a new master's degree program. R I Med J. 2015;98(9):22-6. Available from: http://www.rimed.org/ rimedicaljournal/2015/09/2015-09-22-pcpm-mello.pdf.
In summary, the Alpert Medical School of Brown University has a culture of strong academic achievement and support, a significant history of and ongoing passion for innovation in medical education, and an accomplished, committed, and engaged medical student body.

4. Epstein-Lubow G, Cineas S, Yess J, Anthony D, Fagan M, George P. Development of a longitudinal integrated clerkship at The Warren Alpert Medical School of Brown University. R I Med J (2013). 2015;98(9):27-31. Available from: http://www.rimed. org/rimedicaljournal/2015/09/2015-09-27-pcpmepstein-lubow.pdf.

5. White J, Riese A, Clyne B, Vanvleet MW, George P. Integrating population and clinical medicine: a new third-year curriculum to prepare medical students for the care of individuals, panels, and population. R I Med J (2013). 2015;98(9):32-5.

6. Clyne B, Rapoza B, George P. Leadership in undergraduate medical education: training future physician leaders. R I Med J (2013). 2015;98(9):36-40. 